\title{
TU/e EmonONEN

\section{Using computational steering to explore the parameter space of stability in a suspension}

\section{Citation for published version (APA):}

Hecht, M., \& Harting, J. D. R. (2009). Using computational steering to explore the parameter space of stability in a suspension. In W. E. Nagel, D. B. Kröner, \& M. M. Resch (Eds.), High Performance Computing in Science and Engineering '09 (pp. 33-48). Springer. https://doi.org/10.1007/978-3-642-04665-0_3

DOI:

10.1007/978-3-642-04665-0_3

Document status and date:

Published: 01/01/2009

\section{Document Version:}

Publisher's PDF, also known as Version of Record (includes final page, issue and volume numbers)

\section{Please check the document version of this publication:}

- A submitted manuscript is the version of the article upon submission and before peer-review. There can be important differences between the submitted version and the official published version of record. People interested in the research are advised to contact the author for the final version of the publication, or visit the $\mathrm{DOI}$ to the publisher's website.

- The final author version and the galley proof are versions of the publication after peer review.

- The final published version features the final layout of the paper including the volume, issue and page numbers.

Link to publication

\section{General rights}

Copyright and moral rights for the publications made accessible in the public portal are retained by the authors and/or other copyright owners and it is a condition of accessing publications that users recognise and abide by the legal requirements associated with these rights.

- Users may download and print one copy of any publication from the public portal for the purpose of private study or research.

- You may not further distribute the material or use it for any profit-making activity or commercial gain

- You may freely distribute the URL identifying the publication in the public portal.

If the publication is distributed under the terms of Article 25fa of the Dutch Copyright Act, indicated by the "Taverne" license above, please follow below link for the End User Agreement:

www.tue.nl/taverne

Take down policy

If you believe that this document breaches copyright please contact us at:

openaccess@tue.nl

providing details and we will investigate your claim. 


\title{
Using Computational Steering to Explore the Parameter Space of Stability in a Suspension
}

\author{
Martin Hecht and Jens Harting \\ Institut für Computerphysik, Pfaffenwaldring 27, 70569 Stuttgart, Germany
}

Summary. We simulate a suspension of model colloidal particles interacting via DLVO (Derjaguin, Landau, Vervey, Overbeek) potentials. The interaction potentials can be related to experimental conditions, defined by the $p \mathrm{H}$-value, the salt concentration and the volume fraction of solid particles suspended in the acqueous solvent. Depending on these parameters, the system shows different structural properties, including cluster formation, a glass-like repulsive structure, or a stable suspension. To explore the parameter space many simulations are required. In order to reduce the computational effort and data storage requirements, we developed a steering approach to control a running simulation and to detect interesting transitions from one region in the configuration space to another. In this article we describe the implementation of the steering in the simulation program and illustrate its applicability by several example cases.

\section{Introduction}

A very active field in physics is soft matter physics which has gained more and more importance during the last years. It comprises for example complex fluids, biological systems like membranes, solutions of large molecules like proteins, or suspensions of small soft or solid particles, which are commonly called "colloids". This is a very general class of materials which have in common that the particles are of a mesoscopic size, which means, they are so small that Brownian motion plays a considerable role for the behavior of the system. Soft matter has in common that the relevant energies (interaction barriers) are on the order of magnitude of the thermal energy $\left(k_{\mathrm{B}} T\right)$. Since one can find examples for these materials nearly everywhere in everyday life (in medicine, food industry, paintings, glue, blood, ceramics,...), scientific research can be very relevant for practical applications. Therfore, a considerable effort has been invested to describe colloidal suspensions from a theoretical point of view and by simulations $[16,31-33,44]$, as well as to understand the particle-particle interactions $[1,10,11,15,59,60]$ and the phase behavior $[7,8,24,35,58]$. Due to the small size of the particles one is usually interested in collective

W.E. Nagel et al. (eds.), High Performance Computing in Science 
properties of an ensemble of many particles, e.g., the viscosity of a suspension, which is determined by the interactions among the individual particles. There are many ways to manipulate these interactions, e.g., by changing the $p \mathrm{H}$-value or the ionic strength of the suspension $[13,14,19,20,62]$, or by adding polymers $[53,54]$ or nanoparticles [36]. This offers the possibility to control the phase behavior of the colloidal suspension as a whole.

However, it is difficult to give quantitative predictions concerning the influence of individual changes in the details of the interactions, because in soft matter systems, typically several effects are in a subtle interplay. The large number of effects to encounter also implies that the parameter space is of high dimension and one has to perform many different simulations, each of which requiring a lot of computing resources, to gain an understanding of all inter-relationships.

Therefore, it is useful to be able to steer the simulation on-line. Steering in this context can mean changing the interaction potentials or other parameters like an externally applied shear rate. It can also mean going back in the simulation time and following a different path starting from an earlier configuration, both options induced by interaction with the user [6, 45]. The most simple case of simulation steering is just to start data acquisition or to provide output data in a running simulation as a response to a request by the user.

As an example system we have modeled $\mathrm{Al}_{2} \mathrm{O}_{3}$-particles suspended in water. The particles are represented by monodisperse spheres of $0.37 \mu \mathrm{m}$ in diameter. They interact via DLVO (Derjaguin, Landau, Vervey, Overbeek) potentials $[10,60]$ as well as a repulsive force ensuring excluded volume for the particles. This model system has been studied in simulations and experiments in previous works $[17,19-21,41,47]$. In this article we discuss how simulation steering can help to explore the parameter space and which problems may occur when using steering techniques. The results have mostly been published in a proceedings paper [18], but in this article we focus more on the technical details and give a more estensive description of the simulation method. Additionally, we comment on our work in progress, which will be published elsewhere together with our collaborators [51].

In the following section we describe our simulation method. Then we describe the implementation of the steering approach. After that, we discuss some examples of simulations where steering helps to explore the parameter space, but we also highlight the limits of the steering approach in which a steered simulation might lead to different results if compared to a non-steered simulation. Finally, we give a summary of our results.

\section{Simulation Method}

We use a coupled algorithm consisting of a Molecular Dynamics (MD) and Stochastic Rotation Dynamics (SRD) part. The simulation method is de- 
scribed in detail in Refs. [17, 19, 21]. The MD algorithm solves the equations of motion for the colloidal particles, whereas the SRD algorithm is a mesoscopic simulation method to take into account the influence of the fluid solvent: it calculates the hydrodynamic interactions and intrinsically includes Brownian fluctuations.

In the MD part we include effective electrostatic interactions and van der Waals attraction, known as DLVO potentials [10, 60]. The repulsive term results from the surface charge of the suspended particles

$$
V_{\text {Coul }}=\pi \varepsilon_{r} \varepsilon_{0}\left[\frac{2+\kappa d}{1+\kappa d} \cdot \frac{4 k_{\mathrm{B}} T}{z e} \tanh \left(\frac{z e \zeta}{4 k_{\mathrm{B}} T}\right)\right]^{2} \times \frac{d^{2} \mathrm{e}^{-\kappa[r-d]}}{r},
$$

where $d$ denotes the particle diameter, $r$ the distance between the particle centers, $e$ the elementary charge, $T$ the temperature, $k_{\mathrm{B}}$ the Boltzmann constant, and $z$ is the valency of the ions of added salt. $\varepsilon_{0}$ is the permittivity of the vacuum, $\varepsilon_{r}=81$ the relative dielectric constant of the solvent, $\kappa$ the inverse Debye length defined by $\kappa^{2}=8 \pi \ell_{B} I$, with $I$ being the ionic strength and the Bjerrum length $\ell_{B}=7 \AA$. The effective surface potential $\zeta$ of the particles depends on the distinct experimental conditions. Apart from the material and surface structure of the particles it is mainly determined by the $p \mathrm{H}$-value and the ionic strength of the solvent. In our previous work [19] we have developed a charge regulation model to describe these dependencies based on the adsorption and desorption of the so-called charge determining ions. The equillibrium of this process depends on two influences: firstly, the electrostatic potential is determined by the surface charge density, i.e., by the amount of ions currently bound to the colloidal particle, and secondly, the electrostatic potential influences the equillibrium of the adsorption process. The $\zeta$ potential of a charged spherical colloidal particle of radius $R$ immersed in an electrolytic environment of relative dielectric constant $\varepsilon_{r}$ and ionic strength $I$ is given within Debye-Hückel theory [9, 40] by

$$
\zeta=\frac{R \sigma}{\varepsilon_{0} \varepsilon_{r}(1+\kappa R)} .
$$

The second part of the model which describes the equillibrium of the adsorption of protons $\left(\mathrm{H}^{+}\right)$on surface leads to the relation [19]

$$
\frac{\sigma}{e N_{S}}=\frac{\delta \sinh \left(\psi_{N}-\zeta e / k_{\mathrm{B}} T\right)}{1+\delta \cosh \left(\psi_{N}-\zeta e / k_{\mathrm{B}} T\right)}
$$

with the Nernst potential $\psi_{N}:=\ln (10)\left(p \mathrm{H}_{z}-p \mathrm{H}\right)$ and $p \mathrm{H}_{z}$ being the point of neutrally charged colloids, the so-called isoelectric point. The equations (2) and (3) can be solved numerically for $\zeta$ as a function of $p \mathrm{H}$ using an iteration scheme. The number density of surface sites $N_{S}, p \mathrm{H}_{z}$ and the parameter $\delta$ are adjusted by fitting the resulting dependencey $\zeta(p \mathrm{H})$ to experimental data for the specific materials [19]. This calibration procedure is required once for 
a known ionic strength. Afterwards the charge regulation model allows to calculate the $\zeta$-potential, and thus the electrostatic interactions among the particles, for different experimental conditions, i.e., a different $p \mathrm{H}$-value and different ionic strength.

The second term of the DLVO potentials which does not depend on the $p \mathrm{H}$-value or the ionic strength is the attractive van der Waals interaction

$$
V_{\mathrm{VdW}}=-\frac{A_{\mathrm{H}}}{12}\left[\frac{d^{2}}{r^{2}-d^{2}}+\frac{d^{2}}{r^{2}}+2 \ln \left(\frac{r^{2}-d^{2}}{r^{2}}\right)\right] .
$$

$A_{\mathrm{H}}=4.76 \cdot 10^{-20} \mathrm{~J}$ is the Hamaker constant [22]. The attractive contribution $V_{\mathrm{VdW}}$ competes with the repulsive term and is responsible for the cluster formation one can observe for conditions in which the attraction dominates.

Since DLVO theory is based on the assumption of large particle separations, it does not correctly reproduce the primary minimum in the potential, which should appear at particle contact. Therefore, we cut off the DLVO potentials and model the minimum by a parabola as described in Refs. [17, 21]. The only free parameter in this model is the depth of the primary minimum, which in principle can be adjusted to experimental data. However, due to surface roughness and lubrication effects, an exact measurement is very difficult. On the other hand, for the simulation it is sufficient to choose the minimum deep enough so that clusters of particles do not break up at the primary minimum.

Once the forces are calculated, the integration of the equations of motion is accomplished in the MD framework using a velocity verlet algorithm [3, 57],

$$
\begin{aligned}
& \mathbf{x}_{i}(t+\delta t)=\mathbf{x}_{i}(t)+\delta t \mathbf{v}_{i}(t)+\delta t^{2} \frac{\mathbf{F}_{i}(t)}{m} \\
& \mathbf{v}_{i}(t+\delta t)=\mathbf{v}_{i}(t)+\delta t \frac{\mathbf{F}_{i}(t)+\mathbf{F}_{i}(t+\delta t)}{2 m} .
\end{aligned}
$$

To ensure excluded volume of the particles we use a repulsive (Hertzian) potential. Below the resolution of the SRD algorithm short range hydrodynamics is corrected by a lubrication force within the MD framework as explained in Refs. [17, 19, 21].

For the simulation of a fluid solvent, many different simulation methods have been proposed: direct Navier Stokes solvers [28, 29, 52, 61], Stokesian Dynamics (SD) [4, 5, 44], Accelerated Stokesian Dynamics (ASD) [55, 56], pair drag simulations [31], Brownian Dynamics (BD) [22, 23], Lattice Boltzmann (LB) [30, 32-34], and Stochastic Rotation Dynamics (SRD) [21, 27, 42]. These mesoscopic fluid simulation methods have in common that they impose certain approximations to reduce the computational effort. Some of them include thermal noise intrinsically, or it can be included consistently. They scale differently with the number of embedded particles, and the complexity of the algorithm differs largely. In particular, there are big differences in the concepts how to couple the suspended particles to the surrounding fluid. 
We apply the Stochastic Rotation Dynamics method (SRD) introduced by Malevanets and Kapral [37, 38]. It intrinsically contains fluctuations, is easy to implement, and has been shown to be well suitable for simulations of colloidal and polymer suspensions [2, 19, 21, 27, 42, 48, 63]. The method is also known as "Real-coded Lattice Gas" [27] or as "Multi-Particle-Collision Dynamics" (MPCD) $[49,50]$. It is based on so-called fluid particles with continuous positions and velocities. A streaming step and an interaction step are performed alternately. In the streaming step, each particle $i$ is moved according to

$$
\mathbf{r}_{i}(t+\tau)=\mathbf{r}_{i}(t)+\tau \mathbf{v}_{i}(t),
$$

where $\mathbf{r}_{i}(t)$ denotes the position of particle $i$ at time $t$, and $\tau$ is the time step. In the interaction step, the fluid particles are sorted into cubic cells of a regular lattice, and only the particles within the same cell interact with each other according to an artificial interaction. The interaction step is designed to exchange momentum among the particles, but at the same time to conserve total energy and total momentum within each cell, and to be very simple, i.e., computationally cheap. Each cell $j$ is treated independently: first, the mean velocity $\mathbf{u}_{j}\left(t^{\prime}\right)=\frac{1}{N_{j}\left(t^{\prime}\right)} \sum_{i=1}^{N_{j}\left(t^{\prime}\right)} \mathbf{v}_{i}(t)$ in cell $j$ is calculated. $N_{j}\left(t^{\prime}\right)$ is the number of fluid particles contained in cell $j$ at time $t^{\prime}=t+\tau$. Then, the velocities of each fluid particle in this cell are rotated according to

$$
\mathbf{v}_{i}(t+\tau)=\mathbf{u}_{j}\left(t^{\prime}\right)+\boldsymbol{\Omega}_{j}\left(t^{\prime}\right) \cdot\left[\mathbf{v}_{i}(t)-\mathbf{u}_{j}\left(t^{\prime}\right)\right] .
$$

$\boldsymbol{\Omega}_{j}\left(t^{\prime}\right)$ is a rotation matrix, which is independently chosen at random for each time step and each cell. We use rotations about one of the coordinate axes by an angle $\pm \alpha$, with $\alpha$ fixed. The coordinate axis as well as the sign of the rotation are chosen at random, resulting in 6 possible rotation matrices. However, there is a great freedom to choose the rotation matrices. Any set of rotation matrices satisfying the detailed balance for the space of velocity vectors could be used here. To remove anomalies introduced by the regular grid, one can either choose the mean free path to be sufficiently large or shift the whole grid by a random vector once per SRD time step [25, 26].

For the coupling of the SRD and the MD simulation, different methods have been introduced in the literature. Inoue et al. proposed a way to implement no slip boundary conditions on the particle surface [27]. To achieve full slip boundary conditions, Lennard-Jones potentials can be applied for the interaction between the fluid particles and the colloidal particles [38, 43]. A more coarse grained method was originally designed to couple the monomers of a polymer chain to the fluid $[12,39]$, but in our previous work [19-21] we have demonstrated that it can also be applied to colloids, as long as no detailed spatial resolution of the hydrodynamics is required. We use this coupling method in our simulations and describe it shortly in the following.

To couple the colloidal particles to the fluid, they are sorted into the SRD cells and included in the SRD interaction step. The stochastic rotation is performed in momentum space instead of the velocity space to take into account 
the difference of inertia between light fluid and heavy colloidal particles. We have described the simulation method in more detail in Refs. [17, 19, 21].

\section{Simulation Steering}

In this section we describe the technical realization of the steering interface in our simulation code. The program is an object oriented code written in $\mathrm{C}++$. Each object contains virtual routines save() and load(), which write the data of the object to a buffer, or load it from there, respectively. The buffer contains a plain text description of all variables contained in the object including their values. This is similar to the $\mathrm{C}++$ source code one would write to initialize an instance of that class. This plain text format is used in the simulation input file, too. The simulation program can dump its whole data structure in the same format, so that the automatically generated files can be used as checkpoints to restart the simulation from this point. The work flow of a simulation, including the actual MD loop as well as data input/output tasks is described in this manner using specialized "workstepclasses". Each workstep-class provides a specialized work()-routine, which performs different actions depending on the actual class type of the respective object. All specialized workstep-class objects are derived from the same (virtual) workstep-class. One of the workstep-classes is designed to change a specified object by using its save() and load() routine. First, the current values are stored to a temporary buffer, then one or several variables may be overwritten by new values, and finally the object to be modified is loaded again from the temporary buffer. The description of the changes may be read from standard input or from a file, similar to the simulation setup, which is read during the initialization of the simulation. workstep-class objects can also be included into the work flow at later times, or may even be disconnected from the usual work flow and bound to standard UNIX system signals. By default a workstep writing particle positions to standard output and a workstep to change objects getting its buffer from standard input are bound to the SIGTTOU ("terminal output") and SIGTTIN ("terminal input") signal, respectively. This allows to embed the simulation program into a framework of shell scripts which generate the appropriate input, redirect the output to a visualization tool and send the signals according to the user interaction. This can be realized in a client-server fashion, even on different hosts and platforms using appropriate scripts and TCP/IP connections. Another way to realize the steering is to drive the system along predefined trajectories in the parameter space, e.g., to continuously change the $p \mathrm{H}$-value and the ionic strength. In this case a workstep which changes objects is included in the standard work flow and regularly reads its instructions from a so-called steering file: in each time step a new set of parameters for the interaction potentials is read in and when the simulation has reached the end of the predefined trajectory, a 
workstep triggering the end of the simulation and final output procedures is loaded from the steering file and added to the simulation protocol.

\section{Results}

We now apply the steering approach just presented to several example cases of computer simulations and discuss its advantages compared to traditional, non-steered simulations. We highlight some pitfalls resulting from the physical background in the context of steering. This section is subdivided into subsections each of them focusing on a particular example to illustrate the steering approach in practice.

\subsection{Pressure Filtration}

Filtration processes are widely used in industry to separate the solid fraction of a suspension from the solvent. In a detailed study we investigate how the interactions among the particles determine the structure of the so-called filter cake [51], i.e., the solid fraction accumulated at the filter plate. The porosity, and especially the permeability of the filter cake strongly determines the efficiency of the filtration process.

In our model the suspended particles cannot pass the filter, whereas the fluid passes through the filter without resistance in an idealized filtration process. The suspended particles agglomerate in front of the filter and form the filter cake. Since the dynamics of the particles is not only governed by the hydrodynamics of the fluid, but also by their DLVO interaction, the density and the structure of the filter cake depend on the $p \mathrm{H}$-value and the ionic strength [51].

In a preliminary study [18] we check if this dependence can be seen in our simulations. We also study how an equillibration prior to the filtration process influences the structure of the filter cake [51]: if attractive forces are present in dilute suspensions, clusters form, which afterwards aggregate at the filter plane. In our simulations the filter is modeled by a closed boundary condition for the particles, which is fully permeable to the fluid. We use fully periodic boundaries for the fluid and drive the system either by applying a force to the fluid [18] or by moving the boundaries for the particles [51]. In the first case the force is applied to a limited region of the fluid so that the fluid is accelerated downwards and drags the particles with the flow towards the filter plane. In the latter case the pressure acting in the process can be controlled by monitoring the total force exerted to the particles by the boundary condition.

In Fig. 1 we plot the density profile for for several $p H$-values at a constant ionic strength of $I=3 \mathrm{mmol} / \mathrm{l}$ and a constant total volume fraction of $\Phi=5 \%$. To gain good statistics for the structure, large simulations are needed. Together with low volume fractions and long simulation runs until the filter cake has formed the question raises how to handle the output data. 


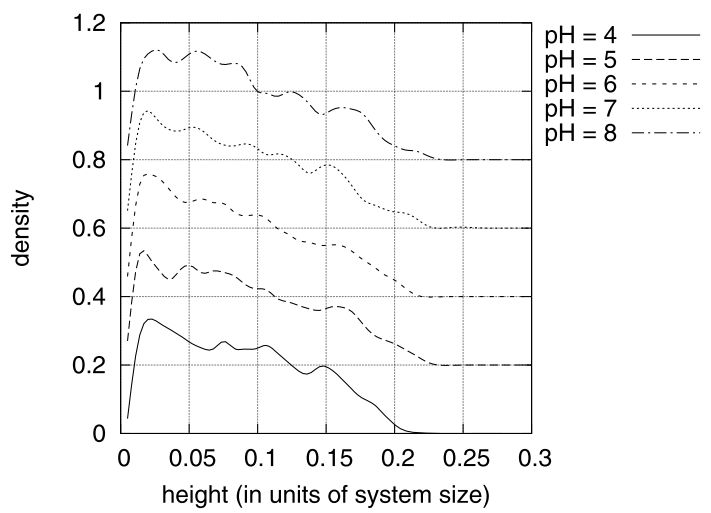

Fig. 1. Local density in terms of solid volume fraction depending on the $p \mathrm{H}$-value (from Ref. [18]). The total volume fraction is kept constant at $\Phi=5 \%$ and the ionic strength at $I=3 \mathrm{mmol} / \mathrm{l}$. The plots are shifted against each other vertically by 0.2 for better visibility. The shape of the profile differs due to the different interactions in each of the cases

Regularly writing the whole configuration and afterwards discarding all configurations in which the simulation has not yet settled is definetly not the optimal procedure. This is a typical problem, in which one would like to observe the running simulation occasionally to check if the filter cake is already formed. When the structure does not change significantly anymore, one would like to measure pressure profiles, local streaming velocities, the shape of the clusters, or simply the final density profile of the filter cake. In this context steering means to initiate data acquisition by user interaction. It is useful to start it manually, when the required conditions are difficult to check automatically. In the present case the density and structure of the filter cake and the time until the filter cake has formed are a priori unknown. This makes it difficult to automatically detect if the desired state has been reached.

\subsection{Observation of Cluster Formation}

Another application of simulation steering is to drive the system along a trajectory in parameter space to explore the regions of certain states or to check if transitions are reversible or not. Especially in soft matter systems, in which the parameter space is very large due to many competing influences, performing such a "steered voyage in parameter space" may help to gain understanding the phase behavior of the system of interest.

In Fig. 2 we show several snapshots of a simulation in which the parameters are constantly varied in this sense of a steered simulation. As indicated in Sec. 3 the trajectory in this simulation was fixed before the simulation was started, but it could as well be delivered to the program "on the fly", such that a steering tool provides the values for the interactions to the program 


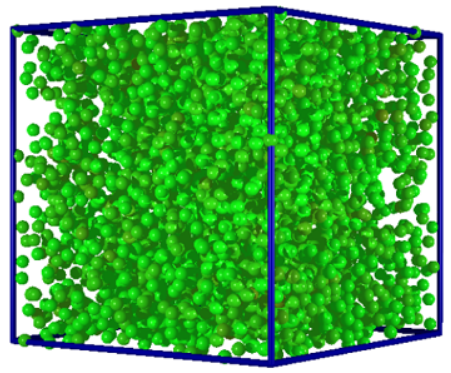

a)

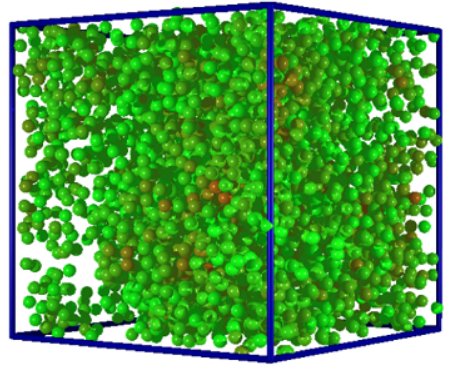

c)

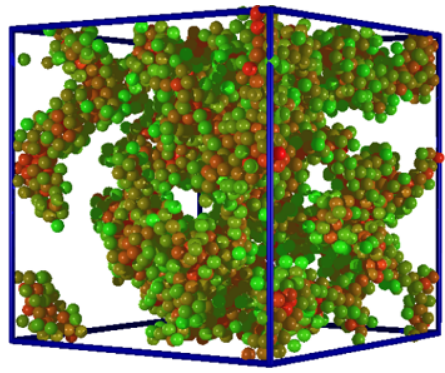

b)

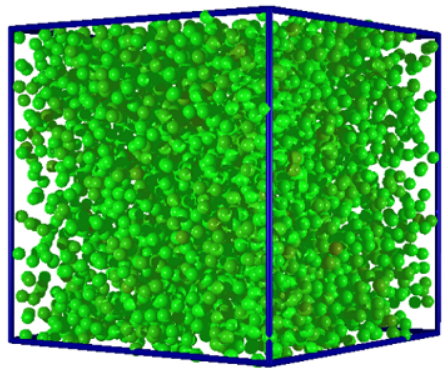

d)

Fig. 2. Selected snapshots during a steered simulation to depict "memory" effects: the color scale denotes the coordination number (Ref. [18]). Particles closer than 2.4 radii are considered to be neighbors. The number of neighbors in this sense is depicted by red color for large numbers, or high local density, and green color for low numbers of neighbors in dilute regions. a) right after initialization, homogeneous distribution of the particles, b) cluster formation after tuning the potentials to be attractive, c) remaining inhomogeneities after steering the simulation back to the region of stabilized suspensions, d) by diffusion the system becomes homogeneous again. The steering path and the points at which the snapshots are taken are illustrated in Fig. 3

each time the user changes them. However, the changes have to be sufficiently smooth, so that it might be necessary to generate intermediate steps.

In Fig. 2 the parameter sub-space of $p H$-value and ionic strength is explored, whereas the volume fraction is kept constant and external driving forces are not applied. In our previous work [17, 20] we have explored the parameter space by performing numerous individual simulations. However, with the newly implemented steering approach one can detect boundaries between different regions of the stability diagram more quickly and more sensitively: When the parameters are modified such that the system crosses the border from a stable suspension (a) to the clustered regime (b), suddenly the system becomes inhomogeneous and cluster formation starts. This inhomogeneity remains (c) when the interactions become repulsive again, but on the charac- 

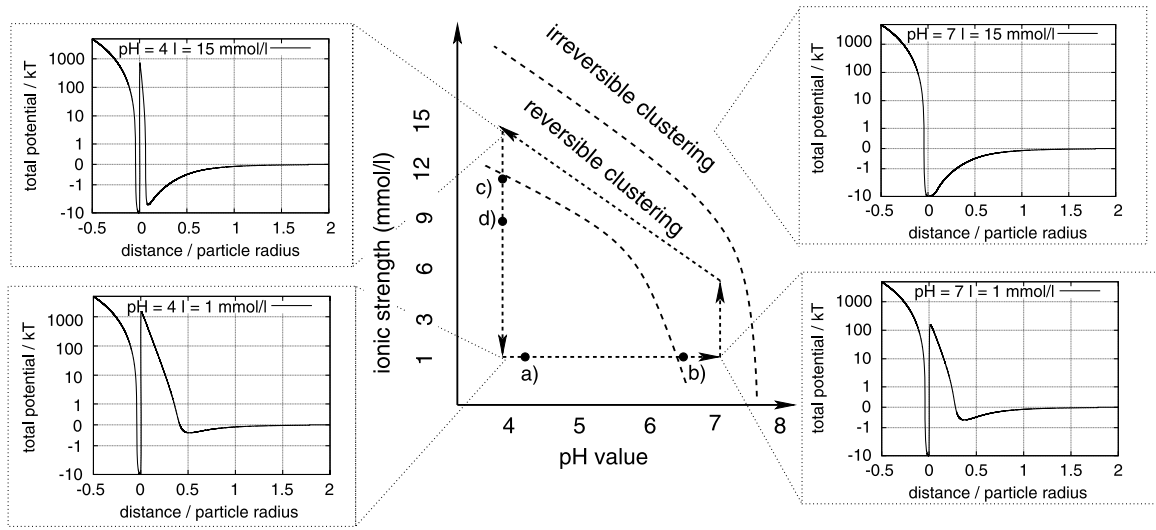

Fig. 3. Trajectory in parameter space for the steering (Ref. [18]): the simulation starts at low $p \mathrm{H}$-value and low ionic strength and follows the dashed path in parameter space. After a short time of equillibration snapshot (a) of Fig. 2 is taken, snapshot (b) after crossing the boundary to the reversibly clustered region, and (c) and (d) after crossing back to the suspended region. The insets show the total potential at some selected extreme points of the parameter space. (see text)

teristic time scale of the diffusion the homogeneity of the system is restored (d).

The steering path in the configuration space is shown in Fig. 3. In the insets of Fig. 3 the effective potential is plotted for several cases. The steering path is chosen such that the cluster formation is reversible and the barrier between the primary and the secondary minimum in the DLVO potential does not go below $5 k_{\mathrm{B}} T$. A case in which the barrier vanishes and irreversible aggregation would appear is also shown in the upper right inset of Fig. 3. The observation in our simulation confirms that the cluster formation is reversible for the chosen trajectory.

However, the simulation "remembers" the trajectory of the steering interaction. Even if the steering path is selected carefully inside the reversible range, the simulated suspension needs a characteristic time to relax after changing the interactions. The interaction can be seen as a perturbation in the physical sense and the system needs time to adopt to the new situation. Using steered simulations one can design and simulate processes in which the interactions are changed by chemical reactions that shift the $p \mathrm{H}$-value. In the steered simulation the response of the simulated system to the perturbation can be observed directly. In any case, a careful interpretation of the simulation data is adviced, because the freedom to change the conditions "on the fly" is bought for the price of having to take into account the response time the system requires to react on the changes. 


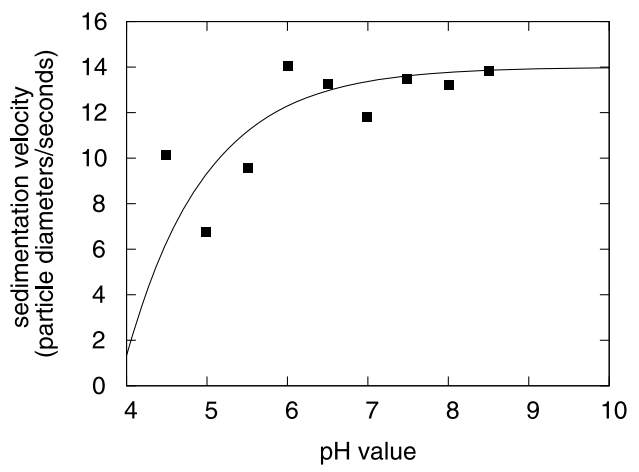

Fig. 4. Sedimentation velocity of a $\Phi=5 \%$ suspension in a closed vessel (Ref. [18]). The sedimentation velocity of the particles in the upper part of the system is averaged over several time steps. The particles at the bottom of the system are not taken into account. The velocity depends on the particle-particle interaction. Clusters settle down faster than individual particles. Symbols denote simulation results, the line is a guide to the eye

\subsection{Sedimentation: Hydrodynamic Interaction}

As we noticed in the previous section, the simulated system may "remember" the steering interaction. Therefore special care is advised when steering a simulation. We illustrate this by another example. Especially, when hydrodynamic interactions are important for the behavior of the system, memory effects become important and might dominate the systems behavior. Then, simulation steering cannot be applied.

Let us consider a sedimenting suspension of particles. If cluster formation is induced by modifying the electrostatic interactions among the particles, the sedimentation proceeds much faster. In Ref. [21] we have confirmed that the sedimentation velocity in our simulation depends on the volume fraction, as it is well-known from sedimentation theory [46]. But even at constant volume fraction we have observed different sedimentation velocities [18] depending on the $p \mathrm{H}$-value: If the particles form clusters, they settle down and the fluid streams around the whole cluster. The resistance is much smaller compared to the case when the particles are distributed homogeneously and the fluid streams around each of them separately (compare Ref. [17] and references therein). In Fig. 4 we have plotted the sedimentation velocity evaluated in several simulations, which only differ by the potentials. The volume fraction is the same for all of them. However, as one can see in the figure, the sedimentation velocity is smaller for low $p \mathrm{H}$-values. Around $p \mathrm{H}=4$ the sedimentation velocity of an isolated particle of 0.6 diameters/s is reached [17] because the interactions among the particles are repulsive for this case and cluster formation is suppressed. This is a typical case in which one should avoid changing the interactions during a simulation, because the behavior of the whole sys- 
tem strongly depends on the distribution of the particles and changes in the interactions are "remembered" by the system in form of correlations due to the hydrodynamic interactions.

\section{Summary}

We have described the implementation of our steering approach in the simulation program and we have illustrated its application in two cases and given an example in which simulation steering is not advisable: if the system is sensitive to correlation effects and contains a "long time memory" which may be the case when hydrodynamic interactions are present, steering should be avoided. On the other hand it can save a large amount of computing time when exploring the parameter space and when the position of transition lines between different regions is investigated. We have shown this by the onset of cluster formation due to modifying the interaction potentials in a model suspension. Steering can be used as well to start data acquisition after a transient at the beginning of a simulation or to adjust the frequency for the data acquisition according to the current state of the simulation. This is especially interesting for large simulations with long transient times, and when a criterion to automatically start data acquisition is difficult to formulate. We have illustrated this by the simulation of filter flow.

Acknowledgments. The High Performance Computing Center Stuttgart and the Scientific Supercomputing Center Karlsruhe are highly acknowledged for providing the computing time and the technical support needed for our research. The authors thank the "Landesstiftung Baden-Württemberg" and the DFG for financial support within the the "Eliteprogram for Postdocs", priority program "nano- and microfluidics", SFB 716, and "Exzellenzakademie Materialwissenschaft und Werkstofftechnik".

\section{References}

1. S. Alexander, P. M. Chaikin, P. Grant, G. J. Morales, P. Pincus, and D. Hone. Charge renormalization, osmotic pressure, and bulk modulus of colloidal crystals: Theory. J. Chem. Phys., 80:5776-5781, 1984.

2. I. Ali, D. Marenduzzo, and J. M. Yeomans. Dynamics of polymer packaging. J. Chem. Phys., 121:8635-8641, 2004.

3. M. P. Allen and D. J. Tildesley. Computer simulation of liquids. Oxford Science Publications. Clarendon Press, Oxford, 1987.

4. J. F. Brady. The rheological behavior of concentrated colloidal suspensions. J. Chem. Phys., 99:567-581, 1993.

5. J. F. Brady and G. Bossis. Stokesian dynamics. Ann. Rev. Fluid Mech., 20:111157, 1988. 
6. J. Chin, J. Harting, S. Jha, P. Coveney, A. Porter, and S. Pickles. Steering in computational science: mesoscale modelling and simulation. Journal of Contemporary Physics, 44:417-434, 2003.

7. D. Costa, P. Hansen, and L. Harnau. Structure and equation of state of interaction site models for disc-shaped lamellar colloids. Mol. Phys., 103:1917-1927, 2005.

8. A. de Candia, E. del del Gado, A. Fierro, N. Sator, and A. Coniglio. Colloidal gelation, percolation and structural arrest. Physica A, 358:239-248, 2005.

9. P. Debye and E. Hückel. Zur theorie der elektrolyte. I. Gefrierpunktserniedrigung. Phys. Z., 24:185, 1923.

10. B. V. Derjaguin and L. D. Landau. Theory of the stability of strongly charged lyophobic sols and of the adhesion of strongly charged particles in solutions of electrolytes. Acta Physicochimica USSR, 14:633, 1941.

11. J. Dobnikar, Y. Chen, R. Rzehak, and H. H. von Grünberg. Many-body interactions in colloidal suspensions. J. Phys.: Condensed Matter, 15:S263, 2003.

12. E. Falck, J. M. Lahtinen, I. Vattulainen, and T. Ala-Nissila. Influence of hydrodynamics on many-particle diffusion in 2 d colloidal suspensions. Eur. Phys. J. E, 13:267-275, 2004.

13. T. J. Graule, F. H. Baader, and L. J. Gauckler. Shaping of ceramic green compacts direct from suspensions by enzyme catalyzed reactions. Ceramic Forum International, 71:314-322, 1994.

14. T. J. Graule, F. H. Baader, and L. J. Gauckler. Casting uniform ceramics with direct coagulation. CHEMTECH, 25(6):31-37, 1995.

15. M. J. Grimson and M. Silbert. A self-consistent theory of the effective interactions in charge-stabilized colloidal dispersions. Molecular Physics, 74:397-404, 1991.

16. L. Harnau and S. Dietrich. Depletion potential in colloidal mixtures of hard spheres and paltelets. Phys. Rev. E, 69:051501, 2004.

17. M. Hecht. Simulation of Peloids. PhD thesis, Universität Stuttgart, Germany, 2007.

18. M. Hecht and J. Harting. Computational steering of cluster formation in Brownian suspensions. Computers and Mathematics with Applications, 58:995-1002, 2009. Proceedings of the Fourth International Conference on Mesoscopic Methods in Engineering and Science, ICMMES (Munich, Germany).

19. M. Hecht, J. Harting, M. Bier, J. Reinshagen, and H. J. Herrmann. Shear viscosity of clay-like colloids in computer simulations and experiments. Phys. Rev. E, 74:021403, 2006.

20. M. Hecht, J. Harting, and H. J. Herrmann. Stability diagram for dense suspensions of model colloidal Al2O3-particles in shear flow. Phys. Rev. E, 75:051404, 2007.

21. M. Hecht, J. Harting, T. Ihle, and H. J. Herrmann. Simulation of claylike colloids. Phys. Rev. E, 72:011408, 2005.

22. M. Hütter. Brownian dynamics simulation of stable and of coagulating colloids in aqueous suspension. PhD thesis, Swiss Federal Institute of Technology Zurich, 1999.

23. M. Hütter. Local structure evolution in particle network formation studied by Brownian dynamics simulation. J. Colloid Interface Sci., 231:337-350, 2000. 
24. A.-P. Hynninen, M. Dijkstra, and R. van Roij. Effect of three-body interactions on the phase behavior of charge-stabilized colloidal suspensions. Phys. Rev. E, 69:061407, 2004.

25. T. Ihle and D. M. Kroll. Stochastic rotation dynamics I: formalism, Galilean invariance, Green-Kubo relations. Phys. Rev. E, 67:066705, 2003.

26. T. Ihle and D. M. Kroll. Stochastic rotation dynamics II: transport coefficients, numerics, long time tails. Phys. Rev. E, 67:066706, 2003.

27. Y. Inoue, Y. Chen, and H. Ohashi. Development of a simulation model for solid objects suspended in a fluctuating fluid. J. Stat. Phys., 107:85-100, 2002.

28. W. Kalthoff, S. Schwarzer, and H. J. Herrmann. Algorithm for the simulation of particle suspensions with inertia effects. Phys. Rev. E, 56:2234-2242, 1997.

29. W. Kalthoff, S. Schwarzer, G. Ristow, and H. J. Hermann. On the application of a novel algorithm to hydrodynamic diffusion and velocity fluctuations in sedimenting systems. Int. J. Mod. Phys. C, 7:543-561, 1996.

30. A. Komnik, J. Harting, and H. J. Herrmann. Transport phenomena and structuring in shear flow of suspensions near solid walls. Journal of Statistical Mechanics: theory and experiment, P12003, 2004.

31. L. E. Silbert, J. R. Melrose, and R. C. Ball. Colloidal microdynamics: Pair-drag simulations of model-concentrated aggregated systems. Phys. Rev. E, 56:70677077, 1997.

32. A. J. C. Ladd. Numerical simulations of particulate suspensions via a discretized Boltzmann equation. Part 1. Theoretical foundation. J. Fluid Mech., 271:285309, 1994.

33. A. J. C. Ladd. Numerical simulations of particulate suspensions via a discretized Boltzmann equation. Part 2. Numerical results. J. Fluid Mech., 271:311-339, 1994.

34. A. J. C. Ladd and R. Verberg. Lattice-Boltzmann simulations of particle-fluid suspensions. J. Stat. Phys., 104:1191, 2001.

35. Y. Levin, T. Trizac, and L. Bocquet. On the fluid-fluid phase separation in charged-stabilized colloidal suspensions. J. Phys.: Condensed Matter, 15:S3523, 2003.

36. J. Liu and E. Luijten. Stabilization of colloidal suspensions by means of highlycharged nanoparticles. Phys. Rev. Lett., 93:247802, 2004.

37. A. Malevanets and R. Kapral. Mesoscopic model for solvent dynamics. J. Chem. Phys., 110:8605, 1999.

38. A. Malevanets and R. Kapral. Solute molecular dynamics in a mesoscale solvent. J. Chem. Phys., 112:7260, 2000.

39. A. Malevanets and J. M. Yeomans. Dynamics of short polymer chains in solution. Europhys. Lett., 52:231, 2000.

40. D. A. McQuarrie. Statistical mechanics. Univ. Science Books, Sausalito, 2000.

41. R. Oberacker, J. Reinshagen, H. von Both, and M. J. Hoffmann. Ceramic slurries with bimodal particle size distributions: Rheology, suspension structure and behaviour during pressure filtration. In N. C. S. Hirano, G. L. Messing, editor, Ceramic Processing Science VI, volume 112, pages 179-184. Am. Cer. Soc., 2001. ISBN 1574981048.

42. J. T. Padding and A. A. Louis. Hydrodynamic and Brownian fluctuations in sedimenting suspensions. Phys. Rev. Lett., 93:220601, 2004. 
43. J. T. Padding and A. A. Louis. Hydrodynamic interactions and Brownian forces in colloidal suspensions: Coarse-graining over time and length-scales. Phys. Rev. E, 74:031402, 2006.

44. T. N. Phung, J. F. Brady, and G. Bossis. Stokesian dynamics simulation of Brownian suspensions. J. Fluid Mech., 313:181-207, 1996.

45. J. Prins, J. Hermans, G. Mann, L. Nyland, and M. Simons. A virtual environment for steered molecular dynamics. Future Generation Computer Systems, 15:485-495, 1999.

46. J. F. Richardson and W. N. Zaki. Sedimentation and fluidisation: Part 1. Trans. Instn. Chem. Engrs., 32:35-53, 1954.

47. S. Richter and G. Huber. Resonant column experiments with fine-grained model material - evidence of particle surface forces. Granular Matter, 5:121-128, 2003.

48. M. Ripoll, K. Mussawisade, R. G. Winkler, and G. Gompper. Low-Reynoldsnumber hydrodynamics of complex fluids by multi-particle-collision dynamics. Europhys. Lett., 68:106-112, 2004.

49. M. Ripoll, K. Mussawisade, R. G. Winkler, and G. Gompper. Dynamic regimes of fluids simulated by multiparticle-collision dynamics. Phys. Rev. E, 72:016701, 2005.

50. M. Ripoll, R. G. Winkler, and G. Gompper. Star polymers in shear flow. Phys. Rev. Lett., 96:188302, 2006.

51. B. Schäfer, J. Harting, M. Hecht, and H. Nirschl. Agglomeration and filtration of colloidal suspensions with DLVO interactions in simulation and experiment. J. Colloid Interface Sci., 2009. (submitted) arxiv:0907.1551.

52. S. Schwarzer. Sedimentation and flow through porous media: Simulating dynamically coupled discrete and continuum phases. Phys. Rev. E, 52:6461-6475, 1995.

53. S. A. Shah, L. Chen, S. Ramakrishnan, K. S. Schweizer, and C. F. Zukoski. Microstructure of dense colloid-polymer suspensions and gels. J. Phys.: Condensed Matter, 15:4751-4778, 2003.

54. S. A. Shah, S. Ramakrishnan, L. Chen, K. S. Schweizer, and C. F. Zukoski. Scattering studies of the structure of colloid-polymer suspensions and gels. Langmuir, 19:5128-5136, 2003.

55. A. Sierou and J. F. Brady. Accelerated Stokesian dynamics simulations. J. Fluid Mech., 448:115, 2001.

56. A. Sierou and J. F. Brady. Shear-induced self-diffusion in non-colloidal suspensions. J. Fluid Mech., 506:285, 2004.

57. W. C. Swope, H. C. Andersen, P. H. Berens, and K. R. Wilson. A computer simulation method for the calculation of equilibrium constants for the formation of physical clusters of molecules: application to small water clusters. J. Chem. Phys., 76:637-649, 1982.

58. V. Trappe, V. Prasad, L. Cipelletti, P. N. Segre, and D. A. Weitz. Jamming phase diagram for attractive particles. Nature, 411:772-774, 2001.

59. R. van Roij and P. Hansen. Van der Waals-like instability in suspensions of mutually repelling charged colloids. Phys. Rev. Lett., 79:3082-3085, 1997.

60. E. J. W. Vervey and J. T. G. Overbeek. Theory of the Stability of Lyophobic Colloids. Elsevier, Amsterdam, 1948.

61. B. Wachmann, W. Kalthoff, S. Schwarzer, and H. J. Herrmann. Collective drag and sedimentation: comparison of simulation and experiment in two and three dimensions. Granular Matter, 1:75-82, 1998. 
62. G. Wang, P. Sarkar, and P. S. Nicholson. Surface chemistry and rheology of electrostatically (ionically) stabillized alumina suspensions in polar media. J. Am. Ceram. Soc., 82:849-856, 1999.

63. R. G. Winkler, K. Mussawisade, M. Ripoll, and G. Gompper. Rod-like colloids and polymers in shear flow: a multi-particle-collision dynamics study. J. Phys.: Condensed Matter, 16:S3941-S3954, 2004. 